10.2478/AMB-2021-0010

\title{
FREQUENCY OF PELVIC LYMPHATIC METASTASES IN PATIENTS WITH CERVICAL CANCER - A RETROSPECTIVE STUDY
}

\author{
E. Chupryna, A. Ganovska, S. Kovachev, S. Gytsova, A. Stoicheva \\ Clinic of General and Oncological Gynecology, Military Medical Academy - Sofia, Bulgaria
}

\begin{abstract}
Introduction and objective: The aim of this study was to determine the incidence of pelvic lymph metastases in cervical cancer, depending on the biology of the tumor and the new changes in the staging. Material and methods: A retrospective database analysis involving 85 patients with cervical carcinoma for a 5-year period was performed. Various factors were analyzed for the purpose of the study. Results: In $20 \%$ of our patients lymph metastases were found with more frequent locus in the right lymph chains. The average number of metastases was equal to or greater than 2. Moderately differentiated cervical carcinomas metastasized most commonly. The most frequent were metastases in squamous cell carcinoma and in Stage $2 B$ under the TNM classification. After changes in FIGO's classification the presence of positive lymph nodes defined the disease as III stage. Conclusion: Our study confirms that cervical carcinoma metastasizes in the pelvic lymph nodes before it reaches the para-aortic lymph nodes.
\end{abstract}

Key words: cervical carcinoma, lymph metastases, TNM, FIGO

Corresponding author: Elena Chupryna, MD, Clinic of General and Oncological Gynecology, Military Medical Academy, Bg-1606 Sofia, E-mail: leno4karu@yandex.ru

REVISED: 23 October 2019, ACCEPTED: 17 December 2019

\section{INTRODUCTION}

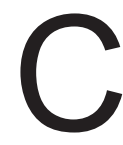
ervical cancer is the fourth most common type of malignant disease that affects women around the world. Almost half a million new cases of the disease are diagnosed each year. After the introduction of cytological screening, the frequency of cervical cancer decreased by more than $40 \%$ over the last 40 years [1].

Direct local invasion of the parametrial tissue and lymphatic dissemination of the regional lymph nodes are the most common types of metastasis in cervical cancer. Metastasis in the para-aortal lymph nodes is characteristic of a progressive pro- cess after phased spreading into pelvic and common iliac lymph nodes. The haematogenous pathway of dissemination is characteristic of advanced low differentiated cancer due to direct invasion of blood vessels. Haematogenous dissemination occurs more often in the venous vessels rather than in the arterial vessels. About $5 \%$ of patients with cervical carcinoma have haematogenous tumor dissemination [2].

The aim of the present study is to investigate the incidence of lymphatic metastasis in cervical cancer and the change in the stage of the disease resulting from them. 


\section{MATERIAL AND METHODS}

A retrospective single-center study was conducted at the Clinic of General and Oncological Gynecology, Military Medical Academy (MMA) for the period 20142018. The study was conducted in accordance with all ethical standards and the principles of the Helsinki Declaration.

For the purpose of this study, data from the electronic system of MMA, history of the disease of each patient, as well as their histological results were collected. Eighty-five (85) women with cervical cancer from T1 to T3, aged 21 to 80 years were included in the study.

Surgical treatment was applied to all patients: trachelectomy was performed in two of the patients and a radical laparo-hysterectomy was done in the rest of the patients. The distribution by type of radical laparo-hysterectomy was classified by Querleu - AbuRustum - Cibula as follows: laparo-hysterectomy type A - in none of the patients, laparo-hysterectomy type B1 - in 4 patients and B2 - in 3, laparo-hysterectomy type $\mathrm{C} 1$ - in 71 patients and $\mathrm{C} 2$ - in 5. Pelvic dissection of the lymph nodes was performed in 75 $(88.3 \%)$ patients and $10(11.7 \%)$ patients had pelvic and para-aortal lymph nodes dissection. The location of surgically removed lymph nodes was described as follows: para-aortic, lymph nodes of the right lymph chain, left lymph node lymph chain.

\section{RESULTS}

We found that the predominant stage of the disease in our target group of patients was $1 \mathrm{~b} 1$ followed by $2 \mathrm{~b}$ in the pTNM classification. The distribution of cervical carcinoma patients by post-operative TNM is shown in Fig. 1.
During the survey $78(92 \%)$ patients were histologically verified to have squamous cell carcinoma of the cervix, of which $1(1.2 \%)$ was verrucous, $12(14.3 \%)$ were non-keratinizing type and $14(16.5 \%)$ were of keratinizing type. In the remaining patients - the subtype of the squamous cell carcinoma was unknown. In $7(8 \%)$ patients histologically verified adenocarcinoma was proven, in $2(2.1 \%)$ cases it was mucinous, in the other $5(5.9 \%)$ no specific subtype was indicated. In our study, we found lymph nodes metastasis in 15 patients with squamous cell carcinoma and in 2 patients with adenocarcinoma.

The average number of lymph nodes removed in each of the 17 patients was 14.6.

After analysis of metastatic lymph nodes of tumors of varying degrees of differentiation, we concluded that in well differentiated carcinomas there was no evidence of metastasis in the lymph nodes. Seven patients $(8.3 \%)$ with low differentiated carcinomas had metastasis in the lymph nodes and $10(11.7 \%)$ patients with moderately differentiated cervical carcinoma had data on lymphatic metastases.

In $17(20 \%)$ out of 85 patients, we found lymphatic metastases distributed in the following locations: lymph nodes metastases in the right pelvic lymph chain - in $8(9.5 \%)$ patients, lymph nodes metastases in the left pelvic lymph chain - in $6(7 \%)$ patients, bilateral metastases in pelvic lymph nodes - at $3(3.5 \%)$ patients. Our study did not detect engagement of para-aortal lymph nodes in oncological processes.

After analysis of the total amount of surgically removed lymph nodes with metastasis, we concluded that the average number of lymphatic metastases, depending on the location, was approximately the same. In the right pelvic lymph node chain the average of lymph nodes with metastasis was 2 , in the left pelvic lymph node chain -2.6 , in the bilateral pelvic lymphatic metastasis -2 .

In this retrospective study, we found the highest occurrence of lymphatic metastasis in Stage $2 b$ in the TNM classification of cervical carcinoma. Metastases in lymph nodes are also present in the smaller stages of the disease starting from 1a1 to $2 \mathrm{a} 2$. The results are presented in Fig 2.

Fig. 1. Distribution of patients by stages 


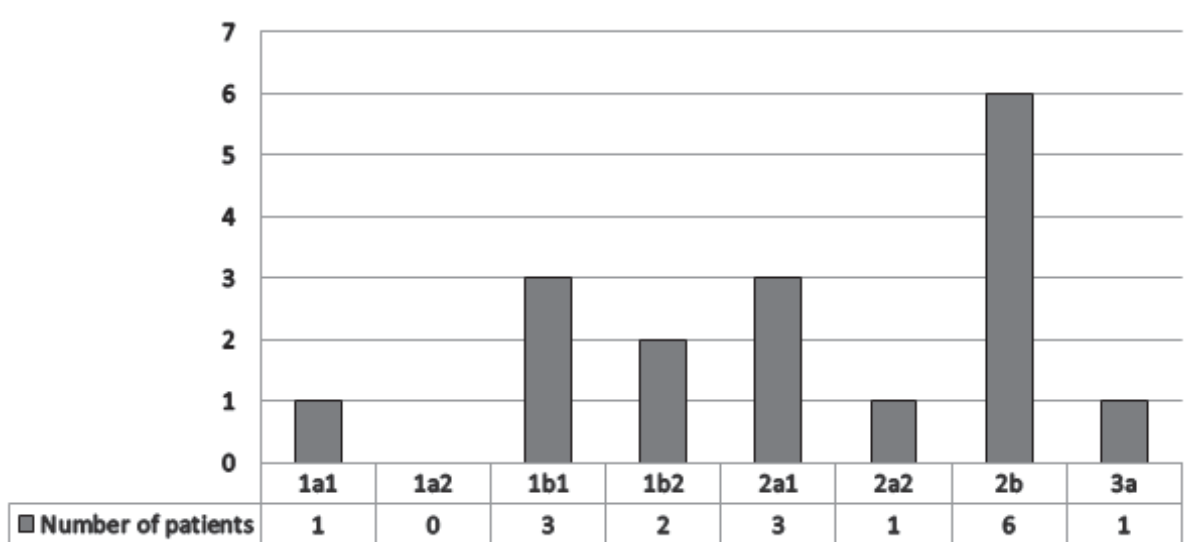

Fig. 2. The distribution of patients with lymph nodes metastases depending on the stage

Following changes in 2018, in the presence of metastases in the lymph nodes, the stage of FIGO's disease is directly defined as IIIC1 in pelvic lymphatic metastases and IIIC2 in para- aortic lymph nodes metastasis, which also changes the approach to these patients and the therapeutic behavior.

\section{DISCUSSION}

Two parallel staging systems exist which determine the stage of cervical carcinoma. Both systems are developed on the basis of clinical rather than surgical criteria. The system of The International Federation of Gynecology and Obstetrics (FIGO) is based on physical examination. In 2018, staging adjustments were made to FIGO classification, with major changes in tumor size and the inclusion of lymphatic metastasis in the staging criteria [3].

Until now, the FIGO staging was based mainly on clinical examination with the addition of certain procedures that were allowed by FIGO to change the staging. In 2018, this has been revised by the FIGO Gynecologic Oncology Committee to allow imaging and pathological findings, where available, to assign the stage [3].

The TNM classification includes a description of tumor size, lymphatic metastasis and the presence of distant metastases. It is divided into two types: clinical (cTNM) and histopathological (pTNM), the latter being administered after the operative treatment [2].

One of the main methods for correct diagnosis and staging of the cervical malignancy is surgical treatment with dissection of the para-aortic and pelvic lymph nodes [4].

Therefore, pathological nodal staging often differs from clinical information and may change the course of treatment. The publication of Marnitz et al describes the results of laparoscopic lymphoid dissection in patients with IB stage cervical cancer. Metastatic lymph nodes are histologically proven in $75 \%$ of these women. In a prospective study by Lim et al the presence of positive para-aortic lymph nodes has been described in almost $8 \%$ of patients with previously made and interpreted as negative results from imaging studies [5].

Even in the early stages of cancer, pelvic lymph node metastases are present. According to the study by Sakuragi et al., the percentage of patients with positive pelvic lymph nodes ranged from 11.5 to $39.2 \%$ in the stages of cervical cancer from IB to IIB. The percentage increases as the disease progresses [6]. In our study, we found the presence of positive lymph nodes most commonly in 2B TNM stage cervical cancer. But we also had one patient with proven stage 1a1 metastases.

A review of 22 articles revealed the presence of paraaortic lymphatic metastases in $18 \%$ of patients in IBIVA FIGO stages. Another source reports that $35 \%$ of stage IIB clinically delivered and $20 \%$ of stage III tumors have metastases to the para-aortic lymph nodes. According to the updated indicators for cervical carcinoma staging, all these cases will be classified as stage IIIC, since lymph node involvement gives a worse prognosis [3].

Squamous cell carcinoma and adenocarcinoma are the two histological subtypes of cervical cancer that are the most common ones. They have different etiology, molecular biology, clinical manifestation, response to therapy and prognosis. In patients with squamous cell carcinoma, the incidence of positive lymph nodes is higher than in those with adenocarcinoma [7].

According to a study by Juan Zhou et al. involving 11830 patients, the number of lymph nodes examined in the squamous cervical carcinoma group is an independent prognostic factor for survival, especially in patients with positive lymph nodes. The team of Pieterse et al. demonstrates that a greater number of excision lymph nodes improve recurrence-free life in the group of patients with histologically proven positive lymph nodes. However, in patients with adenocarcinoma, the number of lymph nodes examined has no prognostic value $[6,7]$. 
Metastasis of cervical carcinoma in the lymph nodes is an important prognostic indicator and the average number of metastatic lymph nodes is critical for the treatment [5].

According to a study by Takeda et al., the survival rate of patients with more than 3 positive lymph nodes over a 5 -year period is about $20 \%$ for IB-IIB stages. Liu $Y$ et al reports a $35 \%$ overall survival of patients with less than two positive lymph nodes and half that percentage with more than two positive lymph nodes in patients with squamous cell carcinoma [6].

Proper assessment in the presence of lymph node metastasis is a main key criterion for an adequate adjuvant and surgical treatment $[4,6]$.

In the research of Monk et al., patients with more than 2 positive lymph nodes had a considerably lower survival rate after radical surgical treatment followed by adjuvant radiotherapy. Tsai et al. have shown that both the number and location of the metastatic lymph nodes affect independently the prognosis of cervical cancer [5].

The presence of more than 2 positive lymph nodes and multiple localizations of pelvic lymph metastases are a criterion for identifying patients with low survival rates [6].

The result of our study shows a more frequent localization of metastatic lymph nodes in the right pelvic lymph chain - in 8 (9.5\%) patients. The Liu Z investigation based on computed tomography images indicates that the presence of engaged lymph nodes is more common in the left lymph node than in the right [8].

Higher tumor grade of cervical cancer is characterized by a larger tumor size, the presence of lymph node metastases, a higher stage of the disease, and age of the patients. The study by Matsuo et al, which included 31,536 women with cervical cancer, divided into three groups based on grade of the tumor, indicates that the higher tumor grade had a worse prognosis for the patient [9].

Patients with lymph node metastases in cervical carcinoma have a higher risk of relapse, lower survival rate and worse prognosis [6].

According to Juan Zhou et al., a larger number of excised lymph nodes reduces the risk of hidden lymph node metastases and improves patient survival, especially in patients with squamous cell carcinoma lymph nodes [7].

Furthermore, it has been proven that aggressive node dissection improves patient survival [5].
According to FIGO recommendations, surgical treatment is suitable for IB1, IB2, and IIA1 patients with advisable intervention volume corresponding to radical hysterectomy type $\mathrm{C}$ with pelvic lymph dissection. In stages IB3 and IIA2 due to the size of the tumor and other risk factors such as metastasis in lymph nodes, involved parametria or engaged by neoplasia surgical margins increase the risk of disease recurrence and therefore the post-operative period, thus it is advisable to administer adjuvant radiation. Another suitable therapeutic option for these stages is concurrent platinum-based chemoradiation [3].

Tumor size, grade and histology type are only few of the factors to determine the stage of cancer. The presence of other different than the standard surgical pathological factors when diagnosing the stage of the disease may cause the results to vary. Our study is retrospective thus carrying the limitations of recall and selection bias.

\section{CONCLUSION}

Knowledge of the behavior of each histological type of cancer, its distribution and metastasis to the tissues are the main parameters that change the behavior of a physician. Further studies are needed to understand the cancer process in order to improve the staging, surgical treatment, radiotherapy, or chemotherapy treatment in the interest of each patient individually and to determine the optimal lymphadenectomy for cervical cancer with different histological subtypes. Changing the classification for staging will lead to a different treatment for some patients.

Our study demonstrates the need to include positive lymph nodes in the staging of the disease and recommends pelvic lymph dissection for patients at high risk for lymphatic metastases based on the characteristics of the cancer. In patients at low risk of lymph node metastasis, it may be possible to consider the option of lymph node preservation in order to avoid the side effects of massive lymphadenectomy.

Our study confirms the claim that cervical carcinoma metastasizes occur in the pelvic lymph nodes before spreading into the paraortal region. Better understanding of how metastasis occur in cancer deceases can help improve the indicators of the overall survival.

According to the new FIGO 2018 data, metastasis in the lymph nodes is reflected in the staging of the disease. Following the introduction of the new FIGO classification, the presence of lymphatic metastases (pelvic and para-aortal) determines the stage of the malignant process that is important for subsequent therapeutic behavior. 
Conflict of interest: All the authors have no conflicts of interest to declare.

Fundings: The study received no funding.

\section{REFERENCES}

1. Yokoi E, Mabuchi S, Takahashi R et al. Impact of histological subtype on survival in patients with locally advanced cervical cancer that were treated with definitive radiotherapy: adenocarcinoma/adenosquamous carcinoma versus squamous cell carcinoma. J Gynecol Oncol. 2017; 28(2): 19.

2. Gallup D. Invasive cervical cancer: Staging and evaluation of lymph nodes. The Spread and Staging of Cervical Cancer. Available on https://www.glowm.com/section_view/heading/ The $\% 20$ Spread $\% 20$ and $\% 20$ Staging $\% 20$ of $\% 20$ Cervical $\% 20$ Cancer/item/231

3. Bhatla N, Aoki D, Sharma D. et al. FIGO Cancer Report 2018 October 2018. Internetional journal of gynecology and obstetrics 2018; 143(2): 22-36.
4. Petignat P. Clinical Review Diagnosis and management of cervical cancer. BMJ 2007; 335:765.

5. Joo J, Kim Y, Nam J. Prognostic significance of lymph node ratio in node-positive cervical cancer patients. Medicine (Baltimore). 2018; 97(30).

6. Liu Y, Zhao L, Li M, Wang J et al. The Number of Positive Pelvic Lymph Nodes and Multiple Groups of Pelvic Lymph Node Metastasis Influence Prognosis in Stage IA-IIB Cervical Squamous Cell Carcinoma. Med J (Engl). 2015; 5; 128(15): 2084-2089.

7. Zhou J, Zhang W, Wu S et al. The impact of examined lymph node count on survival in squamous cell carcinoma and adenocarcinoma of the uterine cervix. Cancer Manag Res. 2017; 9: 315-322.

8. Liu Z, Hu K, Liu A et al. Patterns of lymph node metastasis in locally advanced cervical cancer. Medicine (Baltimore). 2016 Sep;95(39):e4814.

9. Matsuo K, Mandelbaum R, Machida $\mathrm{H}$ et al. Association of tumor differentiation grade and survival of women with squamous cell carcinoma of the uterine cervix. J Gynecol Oncol. 2018 Nov; 29(6): e91. 